\title{
CONHECIMENTO E USO DE PLANTAS MEDICINAIS POR ACADÊMICOS DO CURSO DE FARMÁCIA
}

\section{KNOWLEDGE AND USAGE OF MEDICINAL PLANTS BY ACADEMICS OF THE PHARMACY COURSE}

\author{
Thiago Alves Xavier dos Santos ${ }^{1}$, Maura Ferreira Martins Terra ${ }^{2}$, Karen Brigitte Diaz \\ Magaña $^{3}$, Osvaldo Antunes da Silva ${ }^{4}$, Eurislene Moreira Antunes Damasceno ${ }^{5}$
}

1 - Farmacêutico, mestrando em Produção Animal na Universidade Federal de Minas Gerais, campus Montes Claros, Minas Gerais, Brasil.

2 - Farmacêutica, Montes Claros, Minas, Gerais, Brasil.

3 - Mestranda em Produção Animal na Universidade Federal de Minas Gerais (UFMG), campus Montes Claros, Minas Gerais, Brasil.

4 - Docente nas Faculdades Unidas do Norte de Minas (FUNORTE) e Faculdade Próminas, Montes Claros, MG, Brasil. 5 - Farmacêutica, Mestre em Cuidados Primários, docente na Faculdades Unidas do Norte de Minas (FUNORTE), Montes Claros, Minas Gerais, Brasil

Autor para correspondência: thiagoax.17@gmail.com

\section{RESUMO:}

As plantas medicinais são utilizadas desde os tempos mais remotos pela humanidade como forma de curar diversas doenças e esses conhecimentos são repassados pelas gerações. No Brasil devido ao seu extenso e diversificado bioma, as plantas medicinais sempre foram utilizadas. Diante disto, objetivou-se avaliar o conhecimento e o uso de plantas medicinais por acadêmicos do curso de Farmácia. Trata-se de um estudo de campo, de natureza transversal e quantitativa, com a amostra composta por 132 acadêmicos, de ambos os sexos, na faixa etária de 18 a 50 anos, do curso de graduação em Farmácia de uma instituição privada de ensino superior. Após aprovação pelo Comitê de Ética em Pesquisa, os dados foram coletados por meio de um questionário semiestruturado adaptado, analisados e interpretados através de estatísticas descritivas. A partir dos dados verificouse que as plantas mais utilizadas pelos acadêmicos foram a erva-doce (Pimpinela anisum) $(11,4 \%)$, babosa (Aloe vera) $(9,8 \%)$ e boldo (Peumus boldus) $(9,1 \%)$, com as principais finalidades terapêuticas $27,3 \%$ para gripe, $18,9 \%$ para inflamações e $8,3 \%$ para o estresse. Conclui-se que os acadêmicos são adeptos ao uso de plantas visto a grande quantidade espécies vegetais mencionadas.

Palavras-chave: Plantas medicinais; Fitoterapia; Saúde.

\section{ABSTRACT:}

Medicinal plants have been used since ancient times by humanity as a way of curing various diseases and this knowledge is passed on by generations. In Brazil, due to its extensive and diversified biome, medicinal plants have always been used. In view of this, the objective was to evaluate the knowledge and the use of medicinal plants by academics of the Pharmacy course. This is a cross-sectional and quantitative field study with a sample of 132 students of both sexes in the age group of 18-50 years of the undergraduate course in Pharmacy of a private institution of higher education. After approval by the Research Ethics Committee, the data were collected through an adapted semi structured questionnaire, analyzed and interpreted through descriptive statistics. From the data it was verified that the plants most used by the academics were fennel (Pimpinela anisum) (11,4\%), slug plant (Aloe Vera) 
$(9,8 \%)$ and boldo (Peumus boldus) $(9,1 \%)$, with the main therapeutic purposes being $27,3 \%$ for influenza, $18,9 \%$ for inflammation and $8,3 \%$ for stress. It is concluded that academics are adept at using plants because of the large number of plant species mentioned.

Keywords: Medicinal plants; Phytotherapy; Health.

\section{INTRODUÇÃO}

Desde os tempos mais remotos, o homem busca nos recursos naturais meios para suprirem as suas necessidades de sobrevivência, bem-estar e cura dos seus males (BADKE et al., 2017). As plantas sempre foram utilizadas, principalmente para fins terapêutico, baseado na sabedoria do senso comum e transmitidos de geração para geração (ARNOUS; SANTOS; BEINNER, 2005; OLIVEIRA; MENINI NETO, 2012).

Planta medicinal é aquela que contém um ou mais de um princípio ativo, conferindo-lhe atividade terapêutica e são capazes de aliviar ou curar diversas enfermidades (BRASIL, 2012).

No Brasil, os registros sobre a utilização das plantas medicinais são desde a época do período colonial quando os primeiros médicos vindos de Portugal, devido à escassez de remédios sintetizados na Europa, começaram a observar o uso e a importância dos remédios de origem vegetal que eram utilizados pelos povos indígenas (BRASIL, 2012).

Com o início de processo de urbanização e industrialização no país, o acesso aos medicamentos sintéticos e as poucas comprovações das propriedades farmacológicas das plantas, fez com que o conhecimento tradicional do uso das plantas medicinais fosse considerado um sinônimo de atraso e charlatanismo (SANTOS, 2014).

Porém, resgatar o conhecimento tradicional é de grande significância para valorização da cultura tradicional das comunidades. Além disso, resgatar esse conhecimento popular a respeito do uso das plantas medicinais é inquestionável, visto que o uso desta prática pode se considerar como um dos principais recursos terapêuticos para o tratamento de diversas doenças de muitas comunidades e ainda pelo fato do Brasil possui um extenso e diversificados biomas, considerado como um dos mais ricos do mundo em espécies vegetais com potencial terapêutico (ARNOUS; SANTOS; BEINNER, 2005; PASA, 2011; BASTOS, LOPES, 2012; PAIXÃO et al., 2013). Diante deste exposto, a referida pesquisa tem como objetivo avaliar o conhecimento e o uso de plantas medicinais por acadêmicos do curso de Farmácia.

\section{METODOLOGIA}

Trata-se de um estudo de campo que apresenta natureza transversal e quantitativa. Realizado 
em uma instituição privada de ensino superior da cidade de Montes Claros - MG. O presente município se caracteriza por ser polo de Educação e Saúde na região norte de Minas Gerais, situando-se a $16^{\circ} 44^{\prime} 06^{\prime \prime}$ de latitude sul e $43^{\circ} 51^{\prime} 43^{\prime \prime}$ de longitude oeste e sua população é composta aproximadamente 402.027 habitantes (XAVIER et al., 2019).

A população do estudo foi constituída por 132 acadêmicos do curso de graduação em Farmácia, de ambos os sexos, na faixa etária de 18 a 50 anos, selecionados intencionalmente, regularmente matriculados, que aceitaram a participar voluntariamente e preencheram o Termo de Consentimento Livre e Esclarecido (TCLE).

A coleta de dados foi realizada após a autorização da diretoria da instituição por meio do Termo de Consentimento da Instituição (TCI). O instrumento utilizado para coleta do mesmo foi um questionário semiestruturado, adaptado do estudo de Damasceno (2016), que abordava características do entrevistado como idade, sexo, turno de aula, grau de conhecimento e interesse no uso de plantas medicinais; quais as formas, finalidades terapêuticas e plantas utilizadas. Ainda foi verificado o local onde os participantes adiquiriram e com quem obtiveram conhecimentos uso sobre as plantas medicinais.

A presente pesquisa foi aprovada pelo Comitê de Ética em Pesquisa da Associação Educativa do Brasil - SOEBRAS, sob o parecer de número 1.698.588, sendo respeitados todos os critérios exigidos pela resolução 466/12 do Conselho Nacional de Saúde que normatiza pesquisas em seres humanos.

Os dados coletados foram analisados e interpretados através de estatísticas descritivas com auxílio do programa estatístico Predictive Analytics Software (PASW STATISTIC). Para verificar a associação entre as variáveis categóricas adotou-se o teste Qui-quadrado $(p<0.05)$.

\section{RESULTADOS E DISCUSSÃO}

Os dados sociodemográficos demostram que entre os 132 acadêmicos participantes pesquisa, o sexo feminino teve a maior prevalência $(65,9 \%)$ em relação ao masculino (34,1\%), com faixa etária 18 a 28 anos (78,0\%). Em um estudo de gênero e carreira científica nas universidades federais da região Norte do Brasil, Tavares e Parente (2015) apuraram que o sexo feminino obteve maior predominância nos cursos de graduação com cerca de $53 \%$ dos participantes. Percebe - se com isso que as mulheres estão em busca de qualificação cada vez mais, possuindo a maior parcela das vagas nos cursos de graduação (NOBREGA et al., 2017). 
Tabela 1 - Perfil sociodemográficos dos acadêmicos do curso de Farmácia.

\begin{tabular}{lcc}
\hline VARIÁVEL & $\mathbf{n}$ & $\%$ \\
\hline AMOSTRA & 132 & $100 \%$ \\
SEXO & & \\
Masculino & 45 & $34,1 \%$ \\
Feminino & $87^{*}$ & $65,9 \%$ \\
FAIXA ETÁRIA & & \\
18 a 28 anos & $103^{*}$ & $78,0 \%$ \\
29 a 39 anos & 24 & $18,2 \%$ \\
40 a 50 anos & 5 & $3,8 \%$ \\
TURNO & & \\
Integral & 30 & $22,7 \%$ \\
Noturno & $102^{*}$ & $77,3 \%$ \\
\hline \multicolumn{2}{c}{ Fonte: Dados da pesquisa $(2017)}$.
\end{tabular}

Observou-se que 41,7\% dos acadêmicos participantes da pesquisa consideram o seu grau de conhecimento sobre o uso de plantas medicinais como médio e apenas 3,8\% consideram ter bastante conhecimento (gráfico 1). Estes resultados corroboram com o estudo de Farias et al. (2017), no qual a maior parte (58\%) dos acadêmicos de diversos cursos de graduação entrevistados, concordaram que possuem pouco conhecimento a respeito do tema abordado. Paulino e colaboradores (2011) consideram que o grau de conhecimento sobre plantas medicinais dos acadêmicos são oriundos do conhecimento popular e são adiquiridos dentro de casa.

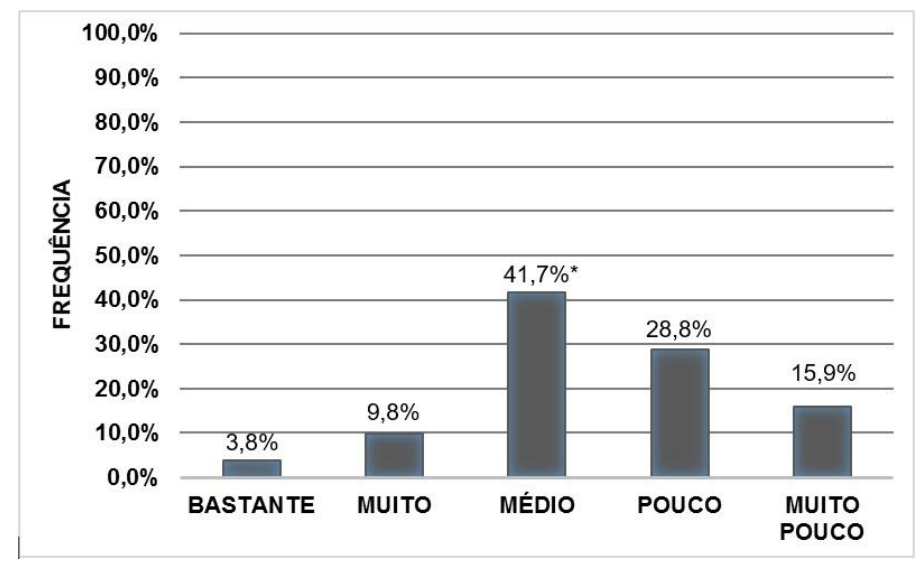

Fonte: Dados da pesquisa (2017)

Gráfico 1 - Grau de conhecimento sobre o uso de plantas medicinais. 
Questionado a respeito do seu interesse sobre plantas medicinais notou-se que a maior parte $(31,8 \%)$ dos acadêmicos consideraram como médio o seu grau de interesse e 27,3\% demostraram ter muito interesse (gráfico 2). Resultados estes inferior aos descritos por Pereira e Malafaia (2011) em que os acadêmicos de Ciências Biológicas, Tecnologia Irrigação e Drenagem e Medicina Veterinária, (85,7\%), $(78,6 \%)(71,4 \%)$, respectivamente, demostram possuirem muito interesse sobre essa temática de plantas medicinais. Segundo a Organização Mundial de Saúde (OMS), nas últimas décadas vem aumentando o interesse da populacão por terapias naturais, gerando aumento no uso de plantas medicinais e medicamentos fitoterápicos (OMS, 2002).

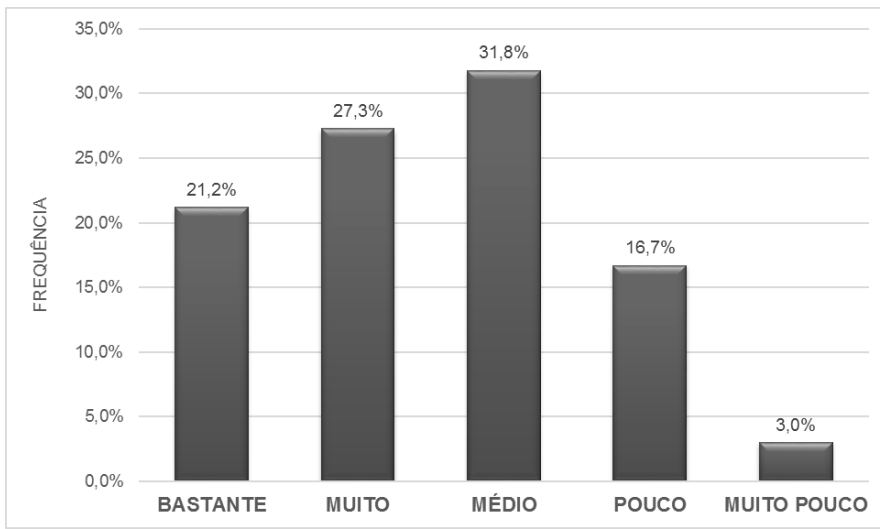

Fonte: Dados da pesquisa (2017).

Gráfico 2 - Grau de interesse sobre plantas medicinais.

Aproximadamente $93 \%$ dos participantes do estudo afirmam que já utilizaram de plantas medicinais para fins terapêuticos e apenas $6,8 \%$ dizem não terem utilizado (tabela 2). Em um estudo com 103 acadêmicos de Farmácia também foi comprovado que $90 \%$ dos entrevistados já utilizaram de plantas medicinais (FARIAS et al., 2017). Desde do inicio da história da huminidade o uso das plantas medicinais desempenha um importante papel nos processos de cura de diversas moléstias, caracterizando-se como um conhecimento tradicional entre a população e este repassado pelas gerações (MAGALHÃES-FRAGA; OLIVIEIRA, 2010: OLIVEIRA; MENINI NETO, 2012).

Dentre as plantas mais utilizadas, a erva-doce (Pimpinela anisum) possui o maior destaque de uso, seguido por babosa (Aloe vera), boldo (Plectranthus barbatus Andrews), arnica (Arnica montana), camomila (Matricaria recitita), hortelã (Menta piperita) e mastruz (Chenopodium ambrosioides) (tabela 2). As plantas mais utilizadas por acadêmicos de Nutrição em Teresina - PI foram o boldo (Plectranthus barbatus Andrews), erva-doce (Pimpinela anisum), hortelã (Menta piperita), camomila (Matricaria recitita), romã (Punica granatum), alho (Allium sativum) (MOURA et al., 2016). Pode - se observar que as plantas citadas no estudo de Moura et al. (2016) não apareceram neste estudo e isso é explicado devido a riqueza de espécies nativas e introduzidas na biodiversidade brasileira, porém mesmo com as diferenças regionais há muita similaridade no uso de plantas medicinais (NÓBREGA et al., 2017). 
Tabela 2 - Uso de plantas medicinais e quais plantas foram usadas.

$\begin{array}{lll}\text { VARIÁVEL } & \text { n } & \%\end{array}$

\section{USO DE PLANTAS MEDICIANAIS}

Sim

$123^{*}$

$93,2 \%$

Não

9

$6,8 \%$

\section{PLANTAS UTILIZADAS}

Alfazema (Lavandula angustifolia)

Aroeira (Schinus terebinthifolius)

Arnica (Arnica montana)

Arruda (Ruta graveolen L.)

Alecrim (Rosmarinus officinalis)

Barbatimão (Stryphnodendreon adstrigens)

Babosa (Aloe vera)

$13 \quad 9,8 \%$

Boldo (Peumus boldus)

Cajueiro (Anacardium occidentale)

1

$0,8 \%$

Cansanção (Cnidosculusurens)

Camomila (Matricaria recitita)

9

$6,8 \%$

Chá verde (Camellia sinensis)

Erva-cidreira (Melissa officinalis L.)

8

$6,1 \%$

Erva-doce(Pimpinela anisum)

Gengibre (Zingiber officinale)

Guaco(Mikania glomerata)

Ginko-biloba(Ginkgo biloba)

Hortelã (Menta piperita)

Mastruz (Chenopodium ambrosioides)

9

$6,8 \%$

Romã (Punica granatum)

Tranchagem (Plantago major) 1

$0,8 \%$

Fonte: Dados da pesquisa (2017) 
No que se diz respeito sobre que finalidade terapêutica as plantas medicinais foram utilizadas pelos acadêmicos, 27,3\% afirmaram que usaram para o tratamento de gripe, 18,9\% para inflamação, 8,3\% para o estresse, 6,7\% para dores estomacais e 5,9\% para diarreia. Em estudo com uma comunidade também na cidade de Montes Claros - MG, destaca-se o tratamento de resfriado como principal finalidade terapêutica (45,8\%), seguido pelo digestivo (12,6\%), cardiovascular (6,8\%) e excretor (2,1\%) (PIRES et al., 2014). Com isso pode-se verificar as diversas enfermidades que são tratadas com o uso de plantas medicinais (PIRES et al., 2014). Porém, as plantas medicinais são usadas também na ausência de doenças, ou seja, em condiçoões normais de saúde, Feitosa et al. (2015) evidenciaram em seu estudo que $50 \%$ dos participantes faziam essa prática.

O chá é a forma de utlização de plantas medicinais mais relatada (VISBISKI; NETO; SANTOS, 2003; CASTRO; SOARES, 2010; PIRES et al., 2014). O chá (49,2\%) também foi evidenciado por estudo, seguido pelo sumo (10,6\%), gargarejo $(6,8 \%)$ e xarope $(5,3 \%)$.

Noventa e oito acadêmicos responderam que aprenderam a utilizar as plantas medicinais com a família, que representa $74,2 \%$, e apenas 3,0\% aprenderam com profissionais da saúde (tabela 3). Resultado similar a outro estudo no qual $71 \%$ dos participantes aprenderam a utilizar as plantas medicinais com a família (MARIANO et al., 2015). O principal motivo para o uso de plantas medicinais é o aprendizado com familiares, isso justificado pela facilidade de propagação das informações entre os membros da família, podendo-se dizer que uso e a manipulação de plantas teve sua origem no contexto familiar (FEITOSA et al., 2015; BADKE et al., 2017).

Para Lima e colaboradores (2014) é indiscutível as vantagens do uso de plantas medicinais para tratamento de enfermidades, porque além de seu baixo custo, apresenta ação biológica eficaz. Contudo os profissionais da saúde devem orientar as pessoas quanto ao uso racional das plantas e esclarecer as dúvidas da população quanto ao uso correto (ARNOUS; SANTOS; BEINNER, 2005).

As plantas medicinais são adquiridas pela maioria dos acadêmicos no quintal de casa $(45,5 \%)$, no mercado (12,9\%), nos quintais de outros (10,6\%) (tabela 3). Em uma pesquisa com pacientes oncológicos no estado de Goiás, Oliveira e colaboradores (2014) constataram que $67,34 \%$ dos pacientes, costumam obter as plantas no próprio quintal de suas residências. Esses estudos divergem de Mariano e colaboradores (2015) em que $70 \%$ dos seus entrevistados disseram que não possuem plantas medicinais no quintal de casa e ainda alegam que a vida urbana que levam e o pouco espaço dos apartamentos e casas dificultam o cultivo.

Os quintais domésticos são considerados como importante fonte de cultivo de espécies medicinais, tais como, hortelã, capim santo, erva babosa, erva cidreira, malva santa e mastruz (AMOROZO, 2002; FARIAS et al., 2017).

O segundo local onde os participantes mais adquiriram as plantas medicinais nesta pesquisa foi o mercado central de Montes Claros - MG. Este local apresenta um forte contexto 
histórico cultural para o município e expressa claramente tradição de uso das plantas medicinais, através das bancas dos raizeiros, que comercializam diferentes tipos de plantas cultivadas no Norte de Minas Gerais (FEITOSA et al., 2015).

Tabela 3 - Como aprendeu e onde adiquire as plantas medicianais.

\begin{tabular}{lll}
\hline VARIÁVEL & N & PORCENTAGEM \\
\hline COMO APRENDEU & & \\
UTILIZAR AS PLANTAS & \\
MEDICINAIS & \\
Família & $98^{*}$ & $74,2 \%$ \\
Vizinhos & 9 & $6,8 \%$ \\
Pastorais & 2 & $1,5 \%$ \\
Raizeiros & 1 & $0,8 \%$ \\
Farmácia & 3 & $2,3 \%$ \\
Profissionais da Saúde & 4 & $3,0 \%$ \\
Faculdade & 6 & $4,5 \%$ \\
Não usam & 9 & $6,8 \%$
\end{tabular}

ONDE ADIQUIREM AS

PLANTAS MEDICINAIS

$\begin{array}{lll}\text { Quintal de casa } & 60^{*} & 45,5 \% \\ \text { Quintal outros } & 14 & 10,6 \% \\ \text { Raizeiros } & 12 & 9,1 \% \\ \text { Farmácia } & 15 & 11,4 \% \\ \text { Vendedor ambulante } & 5 & 3,8 \% \\ \text { Mercado } & 17 & 12,9 \% \\ \text { Não fez uso } & 9 & 6,8 \%\end{array}$

Fonte: Dados da pesquisa (2017)

Sobre a importância de se ter disciplinas sobre plantas medicinais e fitoterapia nos cursos de graduação da área da saúde, 93,2\% responderam que sim, não apresentando muita discrepância com o estudo de Maia (2013) no qual 86,2\% dos acadêmicos de Enfermagem também acham importante. Porém Farias et al. (2017), encontraram um resultado inferior com acadêmicos de Farmácia, em que 62\% acham importante. Segundo Brasil (2006), com o advento da Política Nacional de Práticas Integrativas e Complementares no SUS espera-se que os profissionais que atuam junto ao sistema sejam 
capacitados para quanto ao uso adequado e racional das plantas medicinais e o ambiente acadêmico é o local mais propicio para novas discussões. Visto que, essas discussões geradas favorecem aos futuros profissionais conhecimento para praticarem a medicina tradicional. (FEITOSA et al., 2016).

\section{CONCLUSÕES}

Conclui-se que os acadêmicos possuem conhecimento sobre plantas medicinais e este foi adquirido através do senso comum passado através das gerações. Pode-se concluir ainda que os acadêmicos são adeptos ao uso de plantas visto a grande quantidade de espécies vegetais mencionadas.

\section{REFERÊNCIAS}

AMOROZO, M. C. M. Uso e diversidade de plantas medicinais em Santo Antônio de Leverger, MT, Brasil. Acta Botanica Brasilica, v. 16, n. 2, p. 189-203, 2002.

ARNOUS, A. H.; SANTOS, A. S.; BEINNER, R. P. C. Plantas medicinais de uso caseiro - conhecimento popular e interesse por cultivo comunitário. Revista Espaço para a Saúde, v. 6, n. 2, p. 01-06, 2005.

BADKE, M.; HEISLER, E.; CEOLIN, S.; ANDRADE, A.; BUDÓ, M.; HECK, R. O conhecimento de discentes de enfermagem sobre uso de plantas medicinais como terapia complementar. Revista de Pesquisa: Cuidado é Fundamental, v. 9, n. 2, p. 459-465, 2017.

BASTOS, R. A. A.; LOPES, A. M. C. A; Fitoterapia na Rede Básica de Saúde: o Olhar da Enfermagem. Revista Brasileira de Ciências da Saúde, n. 14, v. 2, p. 21-28, 2010.

BRASIL. Ministério da Saúde. Política Nacional de Plantas Medicinais e Fitoterápicos. Secretaria de Ciência, Tecnologia e Insumos Estratégicos - Departamento de Assistência Farmacêutica. Distrito Federal, 2006.

BRASIL. Ministério da Saúde. Práticas integrativas e complementares: plantas medicinais e fitoterapia na Atenção Básica. Secretaria de Ciência, Tecnologia e Insumos Estratégicos Departamento de Atenção Básica. Distrito Federal, 2012.

CASTRO, L. M.; SOARES, A. F. Aspectos produtivos e o conhecimento sobre fitoterapia por parte de alunos do curso de Farmácia da FAMINAS, Muriaé - MG. Revista Científica da Faminas, v. 6, n. 2, p. 31-45, 2010. 
DAMASCENO, E. M. A. Fitoterapia e Profissionais da Saúde na Estratégia da Saúde da Família. [Dissertação de Mestrado em Cuidado Primário em Saúde]. Montes Claros-MG: Universidade Estadual de Montes Claros, 2016. 76 p.

FARIAS, A. M. B.; VALLATTI, T. B.; OLIVEIRA, A. A.; SALVI, J. O. A fitoterapia entre acadêmicos das Ciências da Vida. Revista Saúde e Desenvolvimento, v.11, n. 9, p. 01 16, 2017.

FEITOSA, M. H. A.; SOARES, L. L.; FERREIRA, I. R.; ANDRADE, M. M.; DIAS, G. P. Plantas medicinais como recurso terapêutico entre funcionários do Centro de Ciências Biológicas e da Saúde da Unimontes. Revista Unimontes Científica, v.17, n. 1, p. 50-59, 2015.

FEITOSA, M. H. A.; SOARES, L. L.; BORGES, G. A.; ANDRADE, M. M.; COSTA, S. M. Inserção do Conteúdo Fitoterapia em Cursos da Área de Saúde. Revista Brasileira de Educação Médica, v. 40, n. 2, p. 197-203, 2016.

LIMA, R. A.; PIRES, S. S.; VIEIRA, N. G. A. A educação ambiental e o uso de plantas medicinais utilizadas pela população do distrito de União Bandeirante - Rondônia. Revista Eletrônica em Gestão, Educação e Tecnologia Ambiental, v. 18, n. 4, p. 1351-1360, 2014.

MAGALHÃES-FRAGA, S. A. P.; OLIVEIRA, M. F. S. Escolas Fitoparceiras: Saúde, Ambiente e Educação através das plantas medicinais. Revista Fitos, v. 5, n. 1, p. 46-58, 2010.

MAIA, M. V. P. O conhecimento dos acadêmicos de Enfermagem a respeito do uso de fitoterápicos. [Monografia de Bacharelado em Enfermagem]. Brasília: Centro Universitário de Brasília, 2013. 16p.

MARIANO, C. R. P.; SOUZA, K. I. L.; FERREIRA, C. L.; PASA, M. C. Uso de plantas medicinais pelos acadêmicos da UFMT. Revista Biodiversidade, v. 14, n. 2, p. 116-124, 2015.

MOURA, A. S. C.; ARAÚJO, L. G.; BRANCO, A. C. S. C.; CARVALHO, L. M. F. Conhecimento sobre plantas medicinais e fitoterápicos: um estudo com acadêmicos de nutrição. Revista Interdisciplinar, v. 9, n. 3, p. 18-25, 2016. 
NÓBREGA, J. S.; SILVA, F. A.; BARROSO, R. F.; CRISPIM, D. L.; OLIVEIRA, C. J. A. Avaliação do conhecimento etnobotânico e popular sobre o uso de plantas medicinais junto a alunos de graduação. Revista Brasileira de Gestão Ambiental, v. 11, n. 1, p. 07-13, 2017.

OLIVEIRA, E. R.; MENINI NETO, L. Levantamento etnobotânico de plantas medicinais utilizadas pelos moradores de povoado de manejo, Lima Duarte-MG. Revista Brasileira de Plantas Medicinais, v. 14, n. 2, p. 311-320, 2012.

OLIVEIRA, L. A. R.; MACHADO, R. D.; RODRIGUES, A. J. L. Levantamento sobre o uso de plantas medicinais com a terapêutica anticâncer por pacientes da Unidade Oncológica de Anápolis. Revista Brasileira de Plantas Medicinais, v. 16, n. 1, p. 32-40, 2014.

OMS. Organización Mundial de la Salud. Estratégias de la OMS sobre medicina tradicional 20022005. Genebra, 2002.67p.

PAIXÃO, J. L. F.; HUMBERTO, D.; OLIVEIRA, J. E. Z. E. Horta orgânica de ervas medicinais: inclusão social na comunidade da Barra em Muriaé/MG - Brasil. Revista Agrogeoambiental, v. 5, n. 2, p.19 - 30, 2013.

PASA, M. C. Saber local e medicina popular: a etnobotânica em Cuiabá, Mato Grosso, Brasil. Boletim do Museu Paraense Emílio Goeldi. Ciências Humanas, v. 6, n.1, p. 179-196, 2011.

PAULINO, R. C.; HENRIQUE, G. P. S. A.; COELHO, M. F. B.; MAIA, S. S. S. Conhecimento sobre plantas medicinais entre alunos da Universidade Federal do Semiárido, Mossoró, RN. Revista Verde, n. 6, v. 4, p. 78-90, 2011.

PEREIRA, I. R; MALAFAIA, G. Conhecimentos sobre plantas medicinais entre estudantes do Instituto Federal Goiano - Campus Urutaí - GO. Revista Saúde e Pesquisa, v. 7, n. 2, p. 275-286, 2014.

PIRES, I. F. B.; SOUZA, A. A.; FEITOSA, M. H. A.; COSTA, S. M. Plantas medicinais como opção terapêutica em comunidade de Montes Claros, Minas Gerais, Brasil, Revista Brasileira de Plantas Medicinais, v. 16, n. 2, p. 426-433, 2014.

SANTOS, L. M. Ecologia de saberes: a experiência do diálogo entre o conhecimento cientifico e o conhecimento tradicional na comunidade quilombola da Rocinha. Tempus Actas de Saúde Coletiva, v. 8, n. 2, p. 243-256, 2014. 
TAVARES, A. S.; PARENTE, T. G. Gênero e carreira acadêmica: Um estudo a partir dos dados das universidades federais da região Norte do Brasil. Revista Ártemis, v.20, n. 2, p. 66-75, 2015.

VISBISKI, V. N.; NETO, P. H. W.; SANTOS, A. L. Uso popular das plantas medicinais no assentamento Guanabara, Imbaú - PR. Publication UEPG: Ciências Exatas e da Terra, Agrárias e Engenharias, v. 9, n. 1, p. 13-20, 2003.

XAVIER, J. L. S.; MENDES, M. M. V.; SANTOS, T. A. X.; BORGES, B. K. A. Conhecimento e utilização de medicamentos genéricos, similares e de referência por pacientes em unidades básicas de saúde de Montes Claros-MG. Revista Uningá, v. 56, n. 1, p. 197-204, 2019. 